\title{
Context-Specific Multi-Site Cingulate Cortical, Limbic Thalamic, and Hippocampal Neuronal Activity during Concurrent Discriminative Approach and Avoidance Training in Rabbits
}

\author{
John H. Freeman, Jr., Cyndi Cuppernell, Karen Flannery, and Michael Gabriel \\ Department of Psychology and Beckman Institute, University of Illinois, Urbana, Illinois 61801
}

\begin{abstract}
This study assessed the context specificity of learning-related neuronal activity: whether the same physical stimuli would elicit different neuronal responses depending on the learning situation. Neuronal activity was recorded simultaneously in six limbic areas as rabbits learned to approach a spout for water reinforcement after a tone $\left(\mathrm{CS}^{+}\right)$and to ignore the spout after a different tone $\left(\mathrm{CS}^{-}\right)$. The rabbits then received avoidance training in which they learned to prevent a foot-shock by stepping in an activity wheel after one tone $\left(\mathrm{CS}^{+}\right)$and to ignore a different tone (CS). Avoidance training sessions were alternated (1 session daily) with sessions in the well learned approach task. The tone assigned as the $\mathrm{CS}^{+}$for approach training was the $\mathrm{CS}^{-}$for avoidance training and vice versa. The neuronal records of the anterior ventral and medial dorsal thalamic nuclei and the anterior and posterior cingulate cortices showed neuronal discrimination appropriate to the approach task during pretraining in the avoidance training apparatus with unpaired presentations of the tones and foot-shock. This finding demonstrated that the discriminative neuronal activity for approach
\end{abstract}

learning was unaffected by a change in context in the pretraining session. However, context-appropriate discrimination occurred in both tasks thereafter, with the exception that medial dorsal thalamic neurons no longer showed discrimination during overtraining in the approach task. Hippocampal area CA1 neurons showed entirely context-appropriate discrimination in both tasks, with no carryover of the approach-relevant discrimination to the avoidance training apparatus. Avoidance training stage-specific peaks of training-induced excitation in different brain areas were not elicited by the same physical stimuli during concurrent approach training sessions. The results are consistent with an involvement of limbic-circuit neuronal activity in the use of context cues for mnemonic retrieval. Differential persistence of the approach-related neuronal discrimination in anterior and posterior cingulate cortex confirmed the previously hypothesized distinct mnemonic functions of these areas.

Key words: context; neuronal activity; discrimination learning; retrieval; memory; limbic system; unit recording
There is currently great interest in the behavioral and neurobiological mechanisms that underlie the influence of background or situational (contextual) stimuli on learning and memory. The importance of contextual stimuli in the governance of memory retricval and conditioned response (CR) performance is well recognized in experimental and cognitive psychology (Tulving and Thomson, 1973; Bouton, 1990; Gordon and Klein, 1994), and contextual stimuli play a central role in neurobiological theories of memory (Hirsh, 1974; Gabriel et al., 1980; Nadel and Willner, 1980; Winocur et al., 1987). Recent experimental data have converged on the conclusion that circuitry of the hippocampal formation is centrally involved in processing of contextual stimuli during Pavlovian and instrumental conditioning (Hirsh, 1974; Winocur et al., 1987; Penick and Solomon, 1991; Phillips and LeDoux, 1992; Kim et al., 1993; McAlonan et al., 1995).

Studies of the neuronal activity accompanying discriminative instrumental learning in rabbits have yielded data of possible relevance to the neural implementation of contextual control. In discriminative avoidance training, rabbits prevent a brief footshock by stepping in a large activity wheel in response to a shock-predictive tone $\left(\mathrm{CS}^{+}\right)$, and they ignore a different tone

\footnotetext{
Received Sept. 18, 1995; revised Nov. 15, 1995; accepted Nov. 22, 1995.

This rescarch was supported by National Institutes of Health Grant NS26736 to M.G.

Correspondence should be addressed to Michael Gabriel, Beckman Institute, University of Illinois, 405 North Mathews Avenue, Urbana, IL 61801.

Copyright (C) 1996 Society for Neuroscience 0270-6474/96/161538-12\$05.00/0
}

$\left(\mathrm{CS}^{-}\right)$that does not predict the shock. Many lesion and recording studies demonstrate involvement in this learning of neurons in sensory and limbic thalamic areas, amygdala, and cingulate cortex (for review, see Gabriel, 1993). Neurons in these areas show excitatory and discriminative training-induced activity (TIA) during learning. Discriminative TIA is the development of significantly greater brief-latency neuronal responses to the $\mathrm{CS}^{+}$than to the $\mathrm{CS}^{-}$, and excitatory TIA is a significant increase in CS-elicited neuronal activity relative to the activity recorded during pretraining with unpaired CS and shock presentations.

The TIA is clearly associative, i.e., it is a product of the pairing of the $\mathrm{CS}^{+}$with the foot-shock and of the $\mathrm{CS}^{-}$with no foot-shock. In addition, certain intriguing properties of the TIA suggest a functional interpretation. The $\mathrm{CS}^{+}$elicits in the aforementioned limbic structures a distinctive topography of excitation. That is, the excitation is nonuniformly distributed with respect to the cytoarchitecture. Remarkably, the cytoarchitectural areas maximally excited by CS presentation change during learning. The progression is the same in all subjects, as demonstrated in average neuronal response data (Gabriel et al., 1991). Moreover, the progression appears to be dependent on projections from the hippocampal formation to the limbic thalamus and cingulate cortex. These projections have been well demonstrated in many studies (Vogt and Gabriel, 1993). Disruption of these efferents via lesions of the subiculum causes a disturbance of the topographic patterns (Gabriel et al., 1987).

The unique topographic patterns at each stage of acquisition 
constitute a neural code signifying the spatio-temporal context that defines the learning situation. That is, a given pattern only occurs in the presence of the $\mathrm{CS}^{+}$presented while the rabbit occupies a particular place (the training apparatus) at a particular time (training stage).

The spatio-temporal code afforded by the topographic patterns may underlie the all-important property of "context specificity" of learned behavior and recall (Gabriel et al., 1991; Gabriel, 1993). We propose that the learned response will be performed with greatest probability only when the appropriate topographic pattern is elicited in the brain, i.e., when the rabbit is in the appropriate spatio-temporal context. Discrimination problems learned at different times and/or in different places will have different topographic patterns. This means that the brain representations of the different problems will be isolated from one another. Each problem will engage a somewhat different neural circuitry. The distinct temporal component of the spatio-temporal code could afford a particularly powerful means of enabling subjects to discriminate between places and events based on when they were last encountered (Chiba et al., 1994).

If this hypothesis is correct, the neuronal activity should be specific to the training context for a particular task. The same physical stimuli should elicit different patterns in the same neurons, depending on the task engaged. To determine whether this happens, neuronal activity was recorded in the aforementioned task-relevant areas as rabbits engaged, concurrently, discriminative instrumental approach and avoidance tasks. Recordings were also made in subfield CA1 of dorsal hippocampus, based on previous findings that hippocampal projections to cingulate cortex and limbic thalamus may be involved in elaboration of the topographic neural code in these other areas.

\section{MATERIALS AND METHODS}

\section{Subjects, electrodes, and surgical procedures}

The subjects were 12 male New Zealand albino rabbits, weighing 1.5-2.0 $\mathrm{kg}$ at the time of delivery by a local supplier. The rabbits were maintained on one cup of rabbit chow daily and were restricted to $150 \mathrm{cc}$ of water/d. They were given at least $5 \mathrm{~d}$ to adjust to this water deprivation before the onset of training.

The rabbits were anesthetized for stereotaxic surgery using a subcutaneous injcction ( $1 \mathrm{mg} / \mathrm{kg}$ body wcight) of a solution containing $60 \mathrm{mg} / \mathrm{ml}$ ketaminc $\mathrm{HCl}$ and $8 \mathrm{mg} / \mathrm{ml}$ xylazine. Six fixed-position microelectrodes fabricated from stainless steel insect pins insulated with Epoxylite were implanted during surgery for chronic postsurgical recording during learning in the behaving animal. Conical recording surfaces were formed by removing insulation (Epoxylite) from the tip of each pin (typical properties: tip lengths, 15-45 $\mu \mathrm{m}$; tip widths at cone base, $15-35 \mu \mathrm{m}$; impedance, $500 \mathrm{k} \Omega$ to $2 \mathrm{M} \Omega$ ). Neuronal activity was monitored acoustically and with an oscilloscope for positioning of the electrodes during implantation. The electrode targets were the anteroventral (AV) nucleus (anterior-posterior, $+2.0 \mathrm{~mm}$ posterior to bregma; medial-lateral, \pm 2.3 ; dorsal-ventral, $7.0-8.0$ ), the mediodorsal (MD) nucleus (anterior-posterior, +4.6 ; medial-lateral, \pm 1.5 ; dorsalventral, 8.0-8.5), the CA1 cell field of Ammon's horn (anterior-posterior, +5.0 ; medial-lateral, \pm 5.0 ; dorsal-ventral, $2.5-3.0$ ), posterior cingulate cortical area $29 \mathrm{c} / \mathrm{d}$ (antcrior-postcrior, 14.0 ; mcdial-latcral, \pm 0.8 ; dorsalventral, $1.0-2.5$ ), and anterior cingulate cortical area $24 \mathrm{~b}$ (anteriorposterior, -2.0 ; medial-lateral, \pm 0.8 ; dorsal-ventral, $2.0-4.0$ ). The stereotaxic coordinates were from the atlas of Girgis and Shih-Chang (1981). Additional information concerning the surgical and recording procedures is available in previous reports (Gabriel et al., 1991).

Collection of neuronal data. During behavioral training, the electrical records were fed into field-effect transistors (FETs), which served as high-impedance source followers located at short distances $(\sim 2.5 \mathrm{~cm})$ from the brain sites of recording. The FET outputs were fed via a shielded cable to single-ended preamplifiers (gain, 40,000; bandwidth, $0.1-10,000 \mathrm{~Hz}$ ) and from there to active band-pass filters (one-half amplitude cutoffs, 600 and $10,000 \mathrm{~Hz}$ ), which removed the slow frequencies while preserving the neuronal action potentials. The filter outputs were fed to Schmitt triggers that produced electronic event-pulses each time one of several multi-unit action potentials exceeded a preset triggering voltage. In addition, the band-pass filter outputs were half-waverectified and integrated following procedures of Buchwald ct al. (1973). The outputs of the integrators were digitized, yielding a measure of integrated multi-unit activity. The time constants for the rise and fall of the integrators were 15 and $75 \mathrm{msec}$, respectively. The Schmitt trigger data provided an index of the discharge frequency of the largest three or four action potentials, whereas the integrated unit activity measured energy fluctuations of the entire record, including activity below the triggering thresholds.

The Schmitt trigger pulses were counted, and integrated unit activity was digitized on each trial (CS presentation) for $1.0 \mathrm{sec}, 0.3 \mathrm{sec}$ before CS onset and $0.7 \mathrm{sec}$ after CS onset. A digital value was stored for each measure and electrode every $10 \mathrm{msec}$ during the sampling interval. The firing frequency counts and integrated unit activity accumulated over trials were averaged to form peristimulus histograms for each training session. Separate histograms were made for $\mathrm{CS}^{+}$and $\mathrm{CS}^{-}$trials, and these were subjected to statistical analysis as described below.

\section{Behavioral training}

One to three weeks after surgery, each rabbit received discriminative approach training in an apparatus designed for instrumental conditioning of head extension and oral contact with a drinking spout. Training was conducted while the rabbits occupied a $61 \times 61 \times 61 \mathrm{~cm}$ aluminum chamber for sound attenuation and electrical shielding. A smaller box, $30 \times 17 \times 37 \mathrm{~cm}$, for partial restraint of movement was located in the chamber and had a mesh floor, mesh sides, and a slot at one end that was large enough for the rabbits to insert their heads to make contact with a drinking spout. A masking noise of $70 \mathrm{~dB}$ (re $20 \mu \mathrm{N} / \mathrm{m}^{2}$ ) was played continuously through a loudspeaker in the chamber. Two pure tones ( 1 or $8 \mathrm{kHz}$; duration, $500 \mathrm{msec} ; 85 \mathrm{~dB}$ re $20 \mu \mathrm{N} / \mathrm{m}^{2}$; rise time, $3 \mathrm{msec}$ ) were assigned in a counterbalanced manner as $\mathrm{CS}^{+}$and $\mathrm{CS}^{-}$. During training, the onset of the $\mathrm{CS}^{+}$was followed after $4 \mathrm{sec}$ by insertion of a drinking spout through the housing wall just in front of the slotted end of the restraining box. The water reward $(3 \mathrm{ml})$ was delivered through the drinking spout in response to electrical grounding that was caused by oral contact with the spout within $6 \mathrm{sec}$ after the onset of the $\mathrm{CS}^{+} . \mathrm{CS}^{-}$ presentation was followed by spout insertion, and spout-contact responses were recorded but water was not presented. The contact responses after $\mathrm{CS}^{+}$and $\mathrm{CS}^{-}$presentations defined the approach $\mathrm{CR}$. The interval between tone presentations was $8,13,18$, or $23 \mathrm{sec}$. These values occurred in a random sequence. Tone presentations were delayed in the presence of electrical artifacts produced by gross movements (e.g., rearing, grooming, and sneezing), and such movements made immediately after tone onset caused discarding and repetition of the trial.

Rabbits received daily training sessions, consisting of 120 trials ( 60 with each CS, presented in a pseudorandom order), until a criterion of discriminative approach learning was reached. The criterion required that the rabbits perform approach CRs on a greater percentage of $\mathrm{CS}^{+}$than on $\mathrm{CS}^{-}$trials. The difference had to be $\supseteq 50 \%$ in two consecutive training sessions. After criterion, discriminative avoidance training was initiated. For this training, the $\mathrm{CS}^{+}$and $\mathrm{CS}^{-}$used for approach training were interchanged. Additional overtraining sessions of approach training alternated with sessions of avoidance training until the criterion of avoidance training plus three additional avoidance overtraining sessions were given.

Avoidance training was administered while the rabbits occupied a rotating wheel apparatus (Brogden and Culler, 1936) located in a different room than for approach training. Masking noise and CS parameters were the same as in approach training, except for the reversal of tone assignments. The onset of the $\mathrm{CS}^{+}$was followed after $5 \mathrm{sec}$ by a foot shock $(1.5-2.5 \mathrm{~mA})$. The foot-shock was terminated by wheel rotation and had an average duration of $\sim 200 \mathrm{msec}$. The rabbits could avoid the foot-shock by producing wheel rotation of at least $2^{n}$ during the $5 \mathrm{sec}$ $\mathrm{CS}$-shock interval. The $\mathrm{CS}^{-}$was not followed by the foot-shock. Rotations after $\mathrm{CS}^{+}$and $\mathrm{CS}^{-}$presentations defined the conditioned avoidance response. Training was continued until criterion was reached, which was determined as for approach training (above) except that the percentage of avoidance responses to the $\mathrm{CS}^{+}$had to exceed the percentage to the $\mathrm{CS}^{-}$by $\geq 60 \%$ rather than $50 \%$. This change was made because of the somewhat lesser magnitudes of discriminative responding found during approach than during avoidance learning. The intertrial intervals were identical to those in the approach task. However, wheel turns during the intertrial interval reset the interval. 


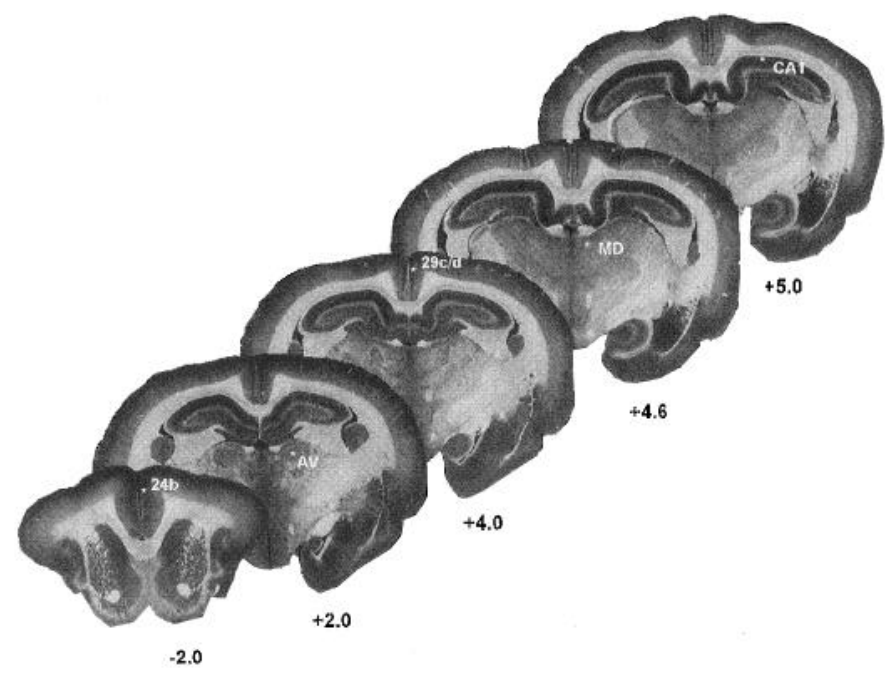

Figure 1. Coronal sections in various planes containing the recording sites, shown by asterisks. From left to right (anterior to posterior) in the figure, the sites are anterior cingulate cortical area $24 b$, the $A V$ thalamic nucleus, posterior cingulate cortical area $29 \mathrm{c} / d$, the $M D$ thalamic nucleus, and hippocampal area $C A 1$. The numbers indicate the approximate anterior-posterior coordinate of the sections (in $\mathrm{mm}$ ) in the stereotaxic atlas of Girgis and Shih-Chang (1981).

Before training in the avoidance and approach tasks, the rabbits received two pretraining sessions. In the first, tones were presented, each 60 times, without reinforcement (i.e., presentation of the water or footshock reinforcer). In the second pretraining session, the tones and the reinforcer were presented in an explicitly unpaired manner. The preliminary training sessions provided baseline data for the assessment of associative changes in behavioral and neuronal responses brought about by pairing of the $\mathrm{CS}^{+}$and reinforcement during the first conditioning session.

\section{Histology}

After training, rabbits were killed via an overdose of sodium pentobarbital followed by transcardial perfusion with normal saline and $10 \%$ formalin. To document the recording sites, the brains were frozen and sectioned at $40 \mu \mathrm{m}$. The sections were photographed while still wet (Fox and Eichman, 1959). After drying, the sections were treated with a metachromatic Nissl and myelin stain using formol-thionin (Donovick, 1974). The recording sites are illustrated in Figure 1.

\section{Data analysis}

The computer-sampled action potential frequency and integrated unit activity values in each of thirty $10 \mathrm{msec}$ intervals after CS onset were normalized with respect to the $300 \mathrm{msec}$ pre-CS baseline period, using the $z$-score transformation. The $z$-scores and behavioral data were submitted to factorial, repeated-measures ANOVA using the P2V program (BMDP Statistical Software, Los Angeles, CA).

\section{RESULTS}

\section{Approach learning}

The rabbits required an average of 7.2 and 12.7 training sessions to attain first significant behavioral discrimination and the acquisition criterion during discriminative approach training, respectively. The first significant discrimination was defined as the session in which the percentage of CRs made to the $\mathrm{CS}^{+}$exceeded the percentage made to the $\mathrm{CS}^{-}$by $\geq 25 \%$. During the session in which this level of performance was reached, the mean percentage of approach CRs performed in response to the $\mathrm{CS}^{+}$was $89.8 \%$ and the mean in response to the $\mathrm{CS}^{-}$was $64.3 \%$. In the session of acquisition in which rabbits reached criterion, the means were 87.8 and $31.8 \%$, respectively. In both instances, discrimination was highly significant (Gabriel et al., 1993).

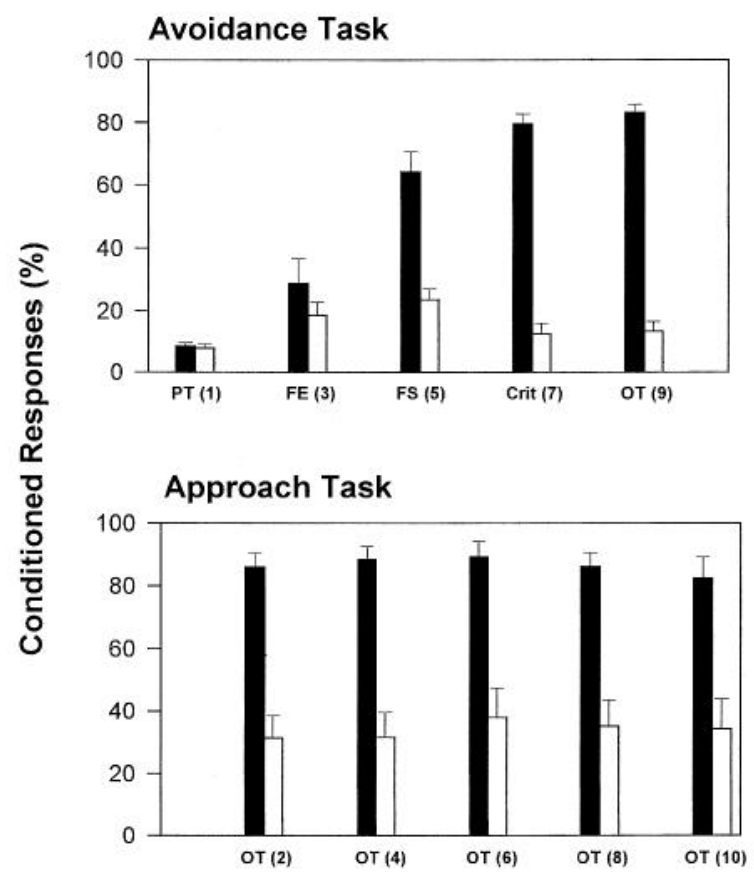

Training Stages

Figure 2. Average percentage of responses after the $\mathrm{CS}^{+}$(solid bars) and $\mathrm{CS}^{-}$(open bars) during discriminative avoidance learning (top graph) and approach training (bottom graph) as a function of training stages. The top graph shows data of the following: pretraining $(P T)$ with explicitly unpaired tone and foot-shock presentations, the session of the first exposure $(F E)$ to conditioning, with paired $\mathrm{CS}^{+}$and foot-shock presentations; the session of the first significant $(F S)$ behavioral discrimination; the session in which the criterion (Crit) of acquisition was attained; and overtraining (OT; sessions of training after criterion). The bottom graph shows data obtained during sessions of overtraining, in which the rabbits performed in the well learned approach task. The numbers in parentheses indicate the temporal order of presentation of the sessions. The top left data of Figures 2-7 were obtained from the pretraining session with tones and reinforcer presentations. These data provide the most relevant baseline for detection of associative changes in the first training session. For brevity, the data of the pretraining session with tones alone are omitted.

\section{Avoidance learning and concurrent approach training}

Avoidance CRs were acquired at essentially the same rate as in many previous studies (for review, see Gabriel, 1993). The mean number of training sessions required for attainment of the first significant discrimination and the acquisition criterion was 2.2 and 4.9 , respectively.

To assess the performance of avoidance responses and concurrent performance of approach responses, the percentage of trials with avoidance and approach CRs, plotted in Figure 2, was submitted to a mixed-factorial, repeated-measures ANOVA with factors of Task (avoidance and approach), Stimulus $\left(\mathrm{CS}^{+}, \mathrm{CS}^{-}\right)$, and Training Stage [represented by data of 5 avoidance training sessions: (1) the session of pretraining with tone and unpaired shock presentations; (2) the first session of avoidance training; (3) the session in which the first significant behavioral discrimination was attained, as defined above; (4) the session in which the acquisition criterion was met; and (5) the third overtraining session presented after attainment of the acquisition criterion]. The training stage factor for approach training was comprised of the five approach overtraining sessions presented on the day before the five stages of avoidance training. 


\section{Nucleus}

\section{Acquisition of Avoidance Task}

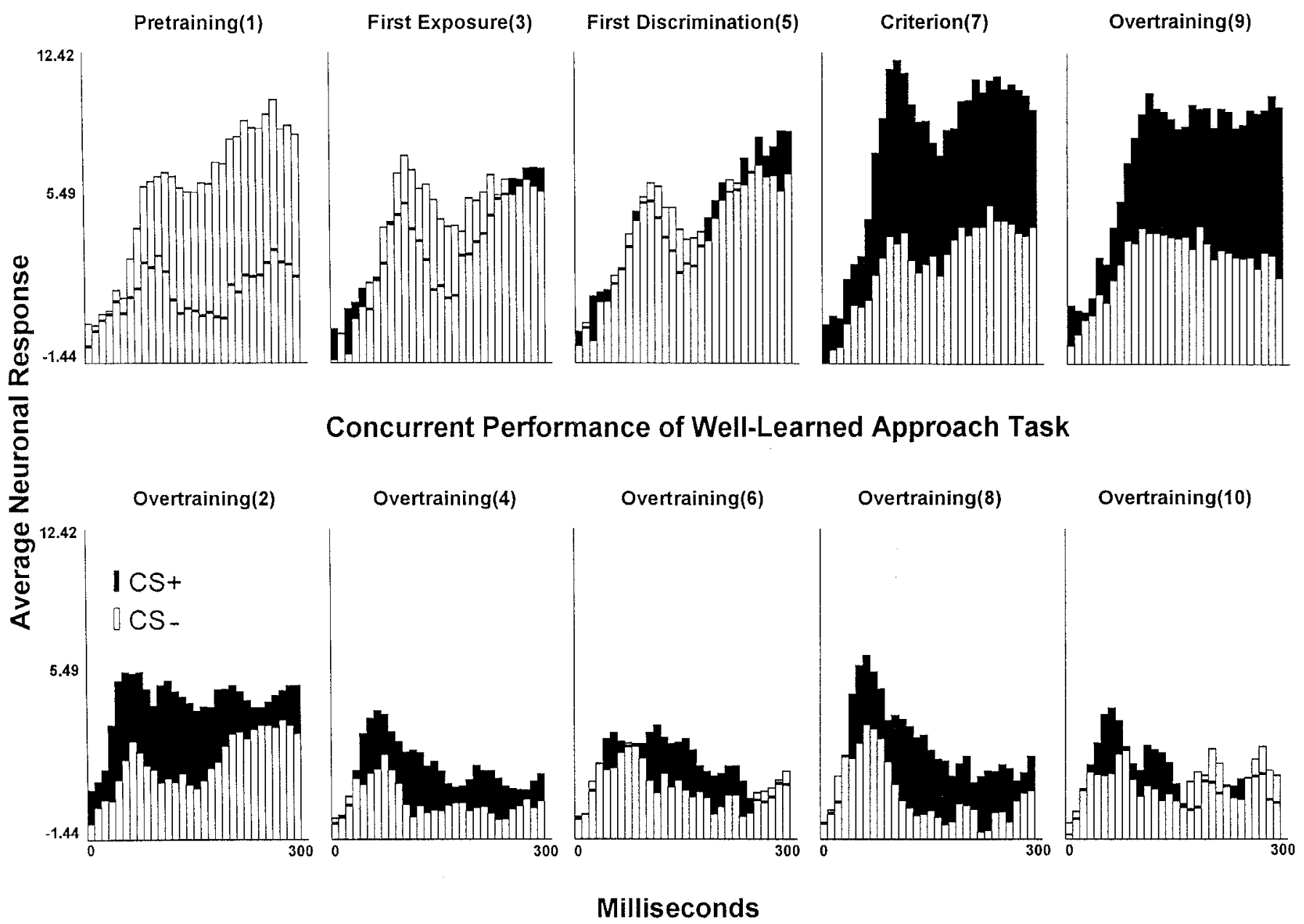

Figure 3. Average integrated unit activity recorded from the MD thalamic nucleus during the stages of avoidance learning (top panels) and concurrent overtraining sessions of approach training (bottom panels). The data in the form of $z$-scores normalized with respect to a $300 \mathrm{msec}$ pre-CS baseline, are shown in 30 consecutive $10 \mathrm{msec}$ intervals after the onset of the $\mathrm{CS}^{+}$(solid bars) and $\mathrm{CS}^{-}$(open bars). See Figure 2 legend for definitions of the avoidance training stages.

The analysis yielded a significant interaction of the Task, Stimulus, and Training Stage factors $\left(F_{(4 / 44)}=29.41, p<0.0001\right)$. Simple effect tests (Winer, 1962) carried out to compare the CR percentages associated with the $\mathrm{CS}^{+}$and $\mathrm{CS}^{-}$presentations at each Task and Training Stage combination showed that significant discrimination (a greater CR percentage in response to the $\mathrm{CS}^{+}$ than to the $\mathrm{CS}^{-}$) was present $(p<0.05)$ during avoidance training stages $3-5$, whereas discrimination was significant $(p<0.01)$ in all five of the concurrent approach training sessions. These results show what is evident in Figure 2-that discriminative responding did not occur during avoidance Training Stages 1 and 2 (preliminary training and the first acquisition session, respectively), but that discriminative acquisition occurred and was expressed during the remaining three avoidance training stages. However, discriminative responding was present during all of the corresponding sessions of training in the well learned approach task. Thus, the rabbits acquired and maintained discriminative performance ap- propriate to the two tasks, despite the reversed assignment of the 1 and $8 \mathrm{kHz}$ tones as $\mathrm{CS}^{+}$and $\mathrm{CS}^{-}$.

The avoidance response performance was virtually identical to levels achieved by rabbits in previous studies, in which only the avoidance task was practiced (Gabriel et al., 1991). (No precedent exists for the performance levels observed in the approach task.) Also, avoidance training did not affect performance of the approach task, because the absolute and discriminative asymptotic performance of the approach response attained at criterion was not altered after introduction of the avoidance task (see Fig, 2).

Neuronal activity: discriminative avoidance learning and concurrent discriminative approach performance Approach learning before avoidance training

All monitored areas showed excitatory and discriminative TIA, and training stage-specific peaks of TIA, during acquisition of the 

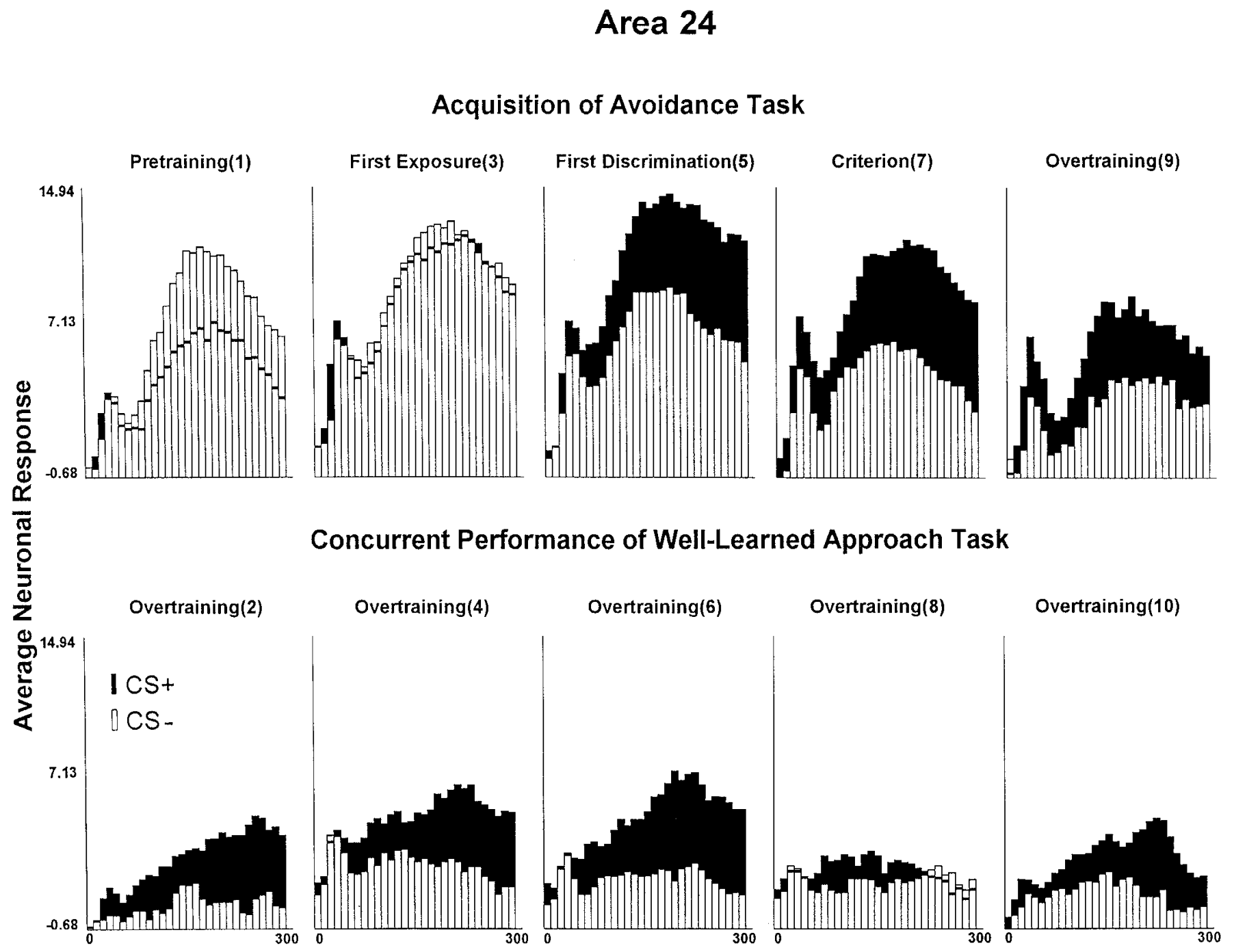

Overtraining(10)

\section{Milliseconds}

Figure 4. Average integrated unit activity recorded in anterior cingulate cortical area $24 \mathrm{~b}$ during the stages of avoidance learning (top panels) and concurrent overtraining sessions of approach training (bottom panels). The data in the form of $z$-scores normalized with respect to a $300 \mathrm{msec}$ pre-CS baseline are shown in 30 consecutive $10 \mathrm{msec}$ intervals after the onset of the $\mathrm{CS}^{+}$(solid bars) and $\mathrm{CS}^{-}$(open bars). See Figure 2 legend for definitions of the avoidance training stages.

discriminative approach response before avoidance training was introduced (Gabriel et al., 1993).

\section{Avoidance learning and concurrent approach performance}

Statistical data. The neuronal data recorded during avoidance learning and in the corresponding approach training sessions, in the form of $z$-scores, were submitted to repeated-measures ANOVA with factors of Task (avoidance or approach), Training Stage (as defined above), Stimulus $\left(\mathrm{CS}^{+}\right.$or $\mathrm{CS}^{-}$), and $10 \mathrm{msec}$ Interval (30 levels).

The cingulate cortical and limbic thalamic neuronal records showed significant discriminative TIA during training (different neuronal activity in response to the $\mathrm{CS}^{+}$than to the $\mathrm{CS}^{-}$). The presence and magnitude of the TIA depended both on the Task and on the Stage of avoidance training at which recordings were made. These conclusions were indicated by simple effect tests carried out after significant interactions of the ' lask, Stimulus, and Training Stage factors of the analysis (Table 1). For certain areas, the interaction was specific to particular sets of post-CS intervals, as indicated by an additional significant interaction of the Task, Stimulus, 'Iraining Stage, and Interval factors, also shown in Table 1. Discrimination between $\mathrm{CS}^{+}$and $\mathrm{CS}^{-}$was indicated for neurons in hippocampal area CA1 by a significant interaction of the Stimulus and Post-CS Interval factors (Table 1). Results of simple effect tests carried out to analyze the significant interactions are reported in the following detailed description of the findings.

Discriminative TIA during preliminary training in the avoidance training apparatus. Significant discriminative TIA appropriate to the already acquired approach task occurred during the preliminary training session (with unpaired presentations of the tones and shock) in the avoidance training apparatus (Figs. 3-6, top left panels). The areas that showed this effect were the MD nucleus, area $24 \mathrm{~b}$ (intervals $13-26 ; p<0.05$ ), the AV nucleus (intervals $16-30 ; p<0.05$ ), and area $29 \mathrm{c} / \mathrm{d}$ (intervals $11-25$ and $27-30 ; p<$ $0.05)$. These results indicate that discriminative TIA in these areas 


\section{AV Nucleus}

\section{Acquisition of Avoidance Task}
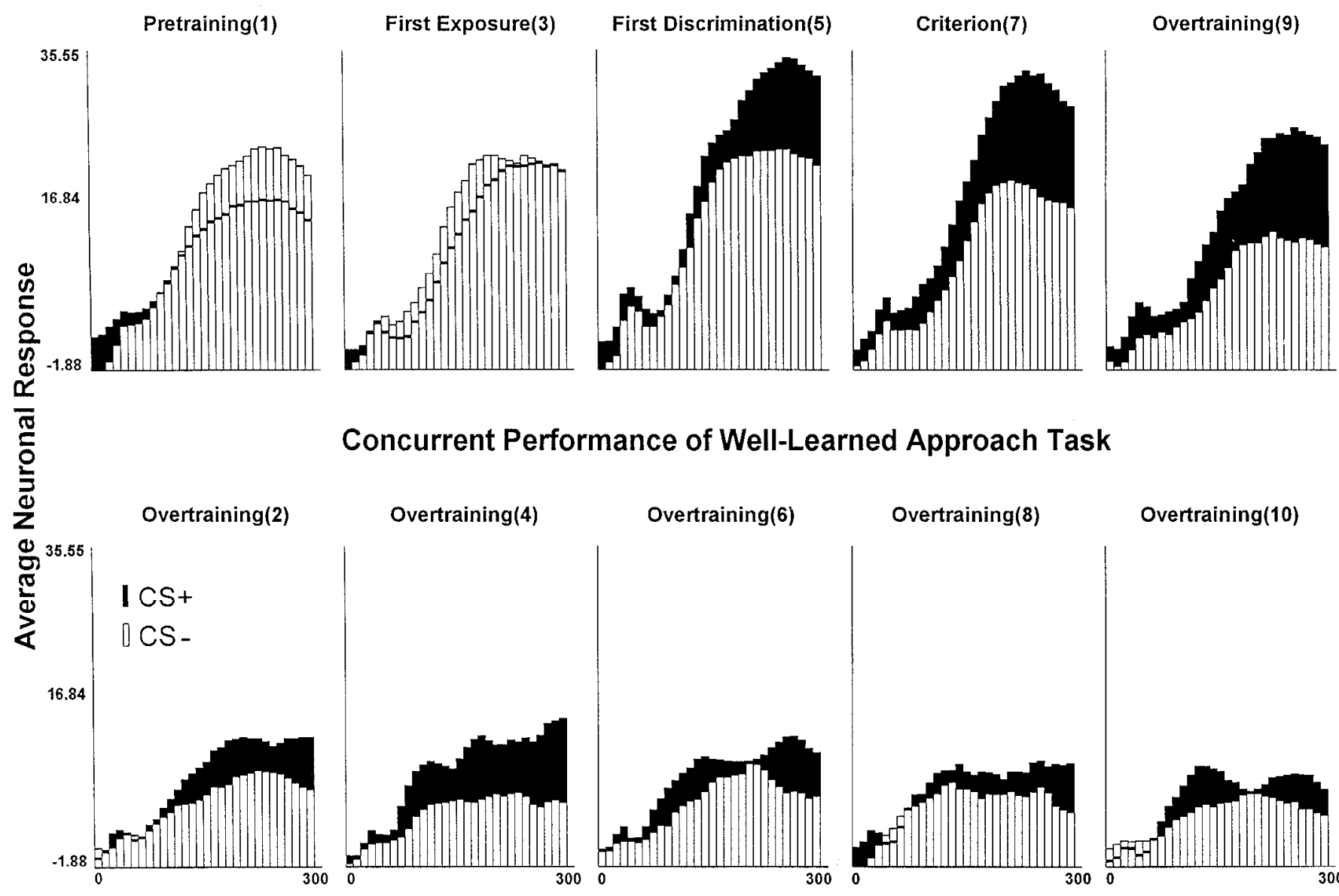

\section{Concurrent Performance of Well-Learned Approach Task}
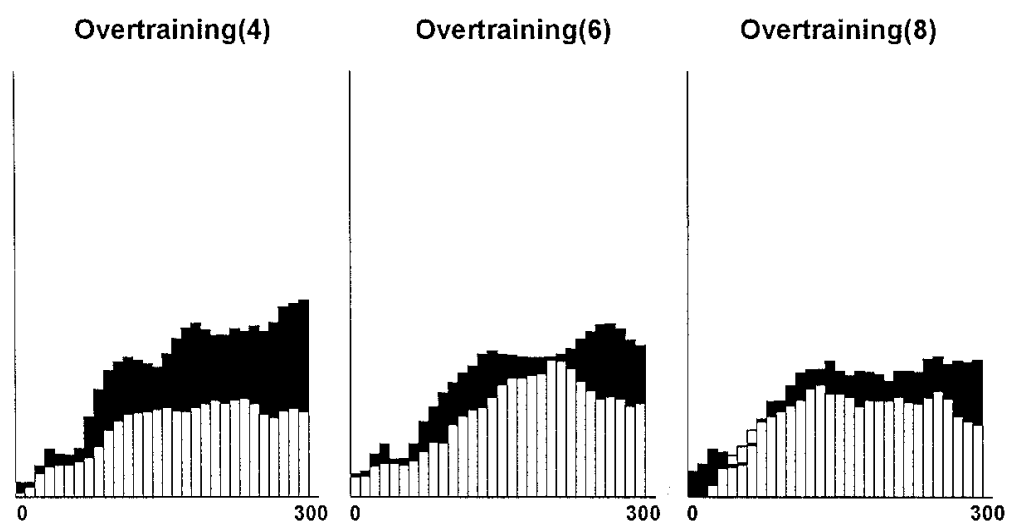

Overtraining(10)

\section{Milliseconds}

Figure 5. Average integrated unit activity recorded from the AV thalamic nucleus during the stages of avoidance learning (top panels) and concurrent overtraining sessions of approach training (bottom panels). The data in the form of $z$-scores normalized with respect to a $300 \mathrm{msec}$ pre-CS baseline are shown in 30 consecutive $10 \mathrm{msec}$ intervals after the onset of the $\mathrm{CS}^{+}$(solid bars) and $\mathrm{CS}^{-}$(open bars). See Figure 2 legend for definitions of avoidance training stagcs.

was not completely dependent on the original training context. Discriminative TIA can be exhibited when the conditioned stimuli are presented in a training context different from the context in which the TIA was originally acquired.

Intriguingly, area CA1 of the dorsal hippocampus was the only area that did not exhibit discriminative TIA during preliminary training in the avoidance learning situation (Fig. 7, top left panel). This absence was not attributable to an absence of approach task coding on the part of CA1 neurons or to a general inability of these records to discriminate. The CA1 records did exhibit significant discriminative TIA during approach training (Gabriel et al., 1993), and the significant interaction of the Stimulus and Interval factors for area CA1 (Table 1) indicated that area CA1 neurons exhibited task-appropriate discriminative TIA throughout avoidance and concurrent approach training (Fig. 7). Thus, neurons of area CA1 were unique in comparison with other areas monitored in not carrying over discriminative TIA, related to approach training, to the avoidance training context during the pretraining session.
Discriminative TIA during avoidance learning. Discriminative TIA appropriate to the avoidance task did not develop in any area before the session of first significant discriminative avoidance behavior (Figs. 3-7). This outcome was uncxpected, because neuronal records of anterior cingulate cortical area $24 \mathrm{~b}$ and the MD nucleus have shown significant discriminative TIA in the first session of training in previous studies (Gabriel, 1993). It is likely that the absence of "first session" discriminative TIA in this study is related to the occurrence of discriminative TIA appropriate to approach training during the pretraining session for avoidance learning. This preexisting TIA was incompatible with avoidance learning-relevant TIA because of the reversal of cue assignment in the two tasks. It is of interest to note that all of the areas that showed the preexisting approach learning-relevant discriminative TIA during preliminary training for avoidance learning lost that discriminative TIA immediately, in the first avoidance training session (compare the first and second top left panels of Figs. 3-6), although this TIA was retained during the corresponding approach training sessions (Figs. 3-7, bottom panels). Therefore, 


\section{Area 29}

\section{Acquisition of Avoidance Task}
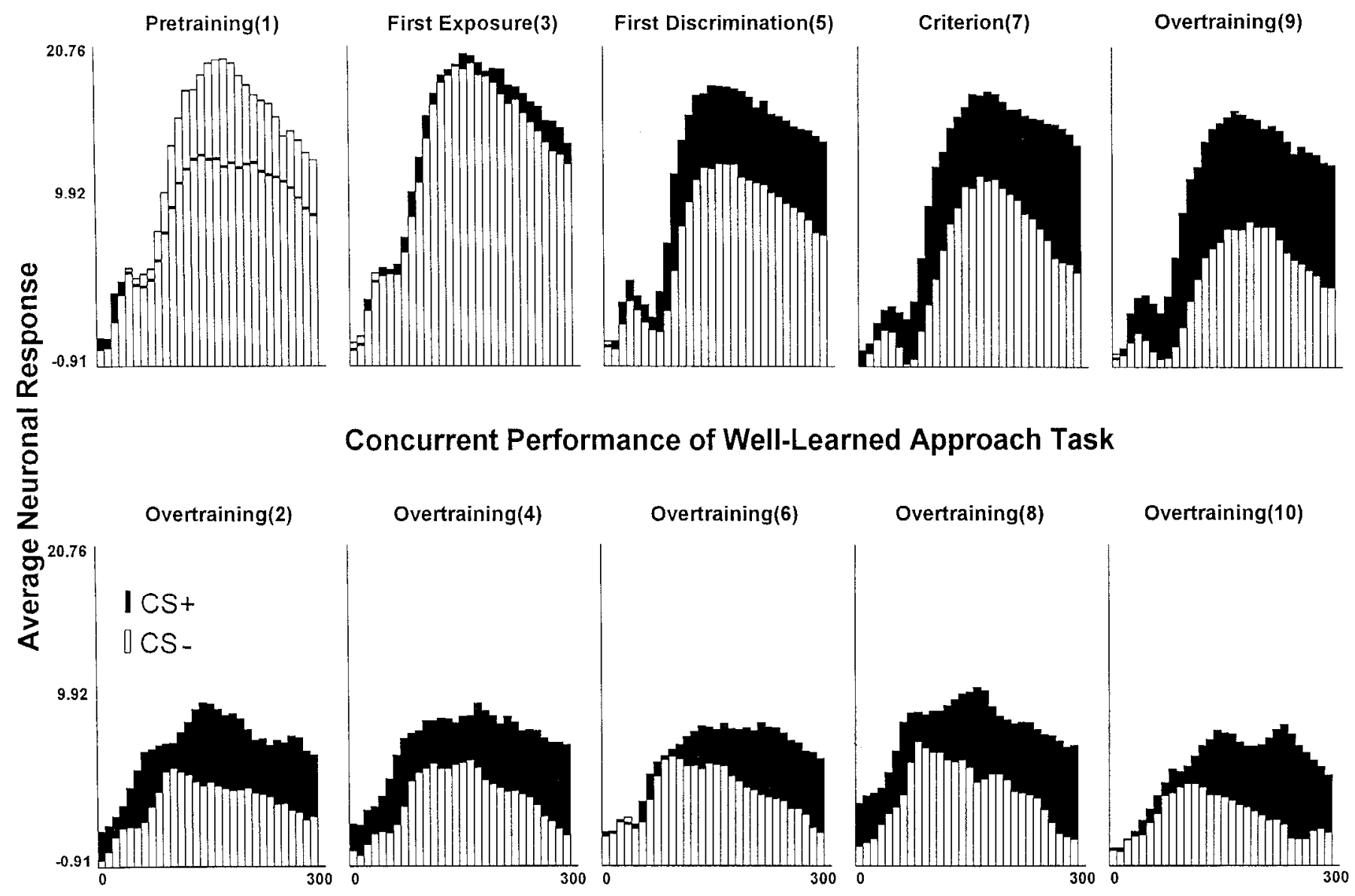

\section{Concurrent Performance of Well-Learned Approach Task}
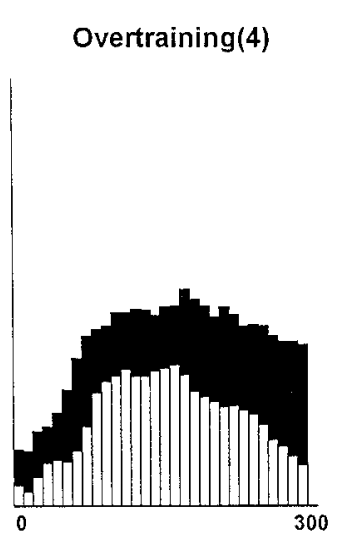

Overtraining(6)

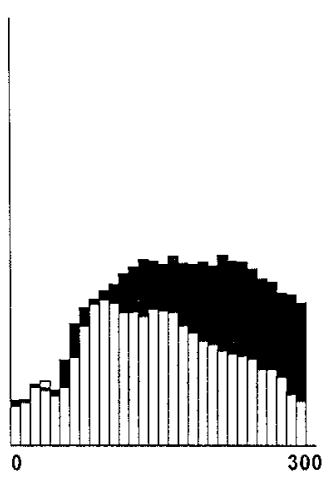

Overtraining(8)

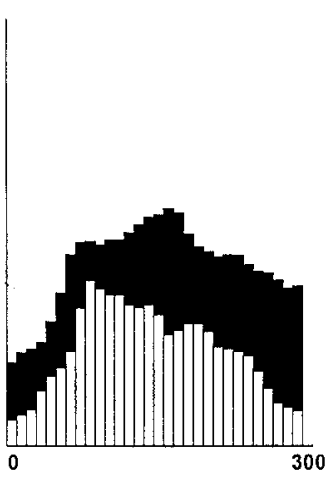

Overtraining(10)

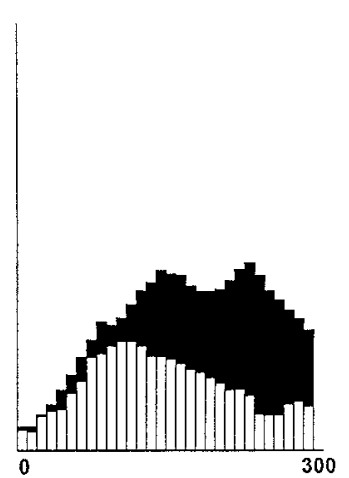

\section{Milliseconds}

Figure 6. Average integrated unit activity recorded in posterior cingulate cortical area $29 \mathrm{c} / \mathrm{d}$ during the stages of avoidance learning (top pancls) and concurrent overtraining sessions of approach training (bottom panels). The data in the form of $z$-scores normalized with respect to a $300 \mathrm{msec}$ pre-CS baseline are shown in 30 consecutive $10 \mathrm{msec}$ intervals after the onset of the $\mathrm{CS}^{+}$(solid bars) and $\mathrm{CS}^{-}$(open bars). See Figure 2 legend for definitions of avoidance training stages.

these areas did show a change in the status of discriminative TIA in the first avoidance training session. Again, the only exception was the neuronal activity of hippocampal area $C \wedge 1$, which showed neither approach training-relevant discriminative TIA during preliminary training for avoidance learning nor any discriminative TIA during the first avoidance training session, while still exhibiting task-appropriate discriminative TIA during other training sessions in both tasks.

The average neuronal records in all of the areas exhibited development of discriminative TIA appropriate to the avoidance task during the course of avoidance acquisition (Figs. 3-7, third through fifth top panels). This discriminative effect occurred in the MD nucleus (Criterion, $p<0.05$; Overtraining, $p<0.05$ ), area 24b (First Discrimination, intervals 10-30, $p<0.05$; Criterion, intervals $12-30, p<0.05$; Overtraining, intervals $12-22$ and $24-$ $29, p<0.05$ ), the AV nucleus (First Discrimination, intervals 13, $15-17$, and $19-30, p<0.05$; Criterion, intervals 10 and $12-30, p<$ 0.05 ; Overtraining, intervals $12-30, p<0.05$ ), area $29 \mathrm{c} / \mathrm{d}$ (First
Discrimination, intervals $9-30, p<0.05$; Criterion, intervals $8-30$, $p<0.05$; Overtraining, intervals $8-30, p<0.05$ ), and area CA1 (across all stages, intervals $1130, p<0.05$ ).

In addition, neuronal records of all areas except the MD nucleus, while exhibiting avoidance related discriminative TIA during avoidance training, also showed discriminative TIA of opposite sign in response to the same physical stimuli, while performing during the interleaved approach training sessions. Neuronal records of posterior cingulate cortical area $29 \mathrm{c} / \mathrm{d}$ and the AV nucleus exhibited significant task-appropriate discriminative TIA in all of the interleaved approach training sessions through the overtraining stage of avoidance training (AV nucleus: first overtraining, intervals $17-22$ and 27-30; second overtraining, intervals $9,10,11-14$, and 16-30; third overtraining, intervals 10 , 14,15 , and 25-30; fourth overtraining, intervals 28-30; fifth overtraining, intervals 12-15-all comparisons, $p<0.05$; Area 29: first overtraining, intervals $6-8,14-21,22$, and $25-30$; second overtraining, intervals $6-8$ and $18-30$; third overtraining, intervals 


\section{Hippocampus (CA1)}

\section{Acquisition of Avoidance Task}

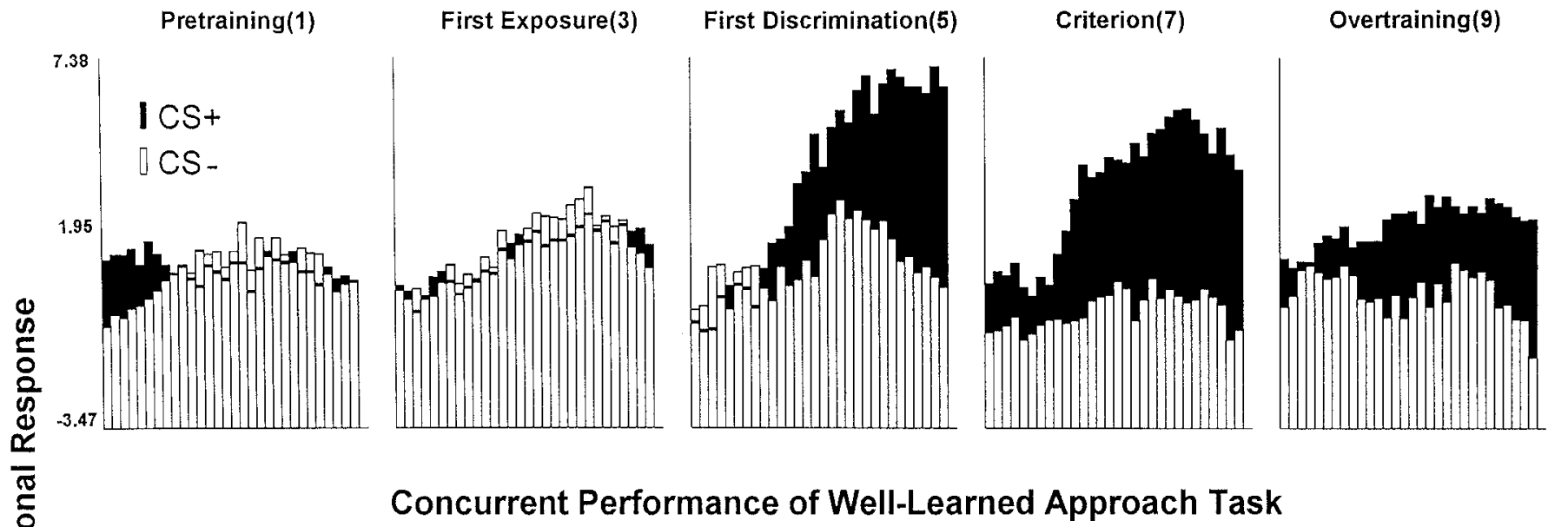

Overtraining(2)

Overtraining(4)

Overtraining(6)

Overtraining(8)

Overtraining(10)
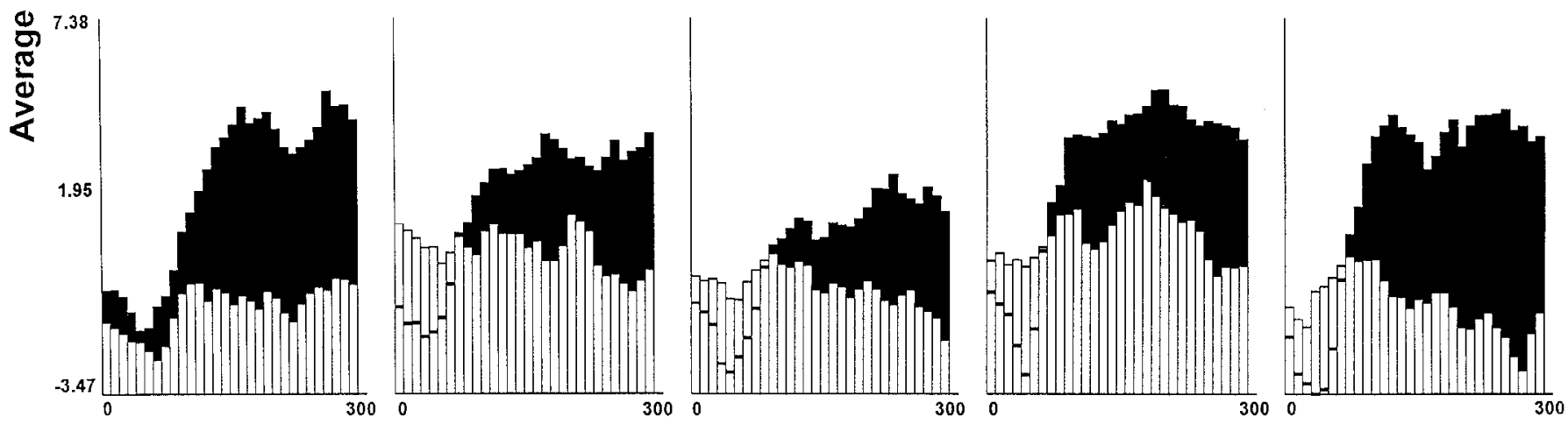

Milliseconds

Figure 7. Average integrated unit activity recorded in hippocampal area CAl during the stages of avoidance learning (top panels) and concurrent overtraining sessions of approach training (bottom panels). The data in the form of $z$-scores normalized with respect to a 300 msec pre-CS baseline are shown in 30 consecutive $10 \mathrm{msec}$ intervals after the onset of the $\mathrm{CS}^{+}$(solid bars) and $\mathrm{CS}^{-}$(open bars). See Figure 2 legend for definitions of avoidance training stages.

Table 1. ANOVA interaction terms used for data analysis

\begin{tabular}{llrl} 
Recording site & Interaction & \multicolumn{1}{l}{} & \multicolumn{1}{l}{} \\
\hline term & $\mathrm{T} \times \mathrm{S} \times \mathrm{C}$ & 5.79 & 0.002 \\
MD & $\mathrm{T} \times \mathrm{S} \times \mathrm{C} \times \mathrm{I}$ & 2.02 & 0.000 \\
Area 24 & $\mathrm{~T} \times \mathrm{S} \times \mathrm{C} \times \mathrm{I}$ & 4.02 & 0.000 \\
AV & $\mathrm{T} \times \mathrm{S} \times \mathrm{C} \times \mathrm{I}$ & 2.52 & 0.000 \\
Area 29 & $\mathrm{C} \times \mathrm{I}$ & 10.80 & 0.000 \\
CA1 & & & \\
\hline
\end{tabular}

T, Task; S, training stage; C, conditional stimuli; I, post-CS intervals.

20-30; fourth overtraining, intervals 6,7 , and $13-30$; fifth overtraining, intervals 14-30-all comparisons, $p<0.05$ ). Neurons in anterior cingulate cortical area $24 \mathrm{~b}$ also exhibited taskappropriate discriminative TIA during approach training, but only in the first (intervals 19-30), second (intervals 19-30), third (intervals 14-30), and fifth (intervals 20-26) sessions of overtrain- ing (all conparisons, $p<0.05$ ). Discriminative TIA was absent in area $24 \mathrm{~b}$ during the approach training session that corresponded to the first discrimination and criterion sessions of avoidance training. The apparent task-appropriate discriminative TIA in the MD thalamic nucleus during the interleaved approach training sessions (Fig. 3) was not significant.

None of the areas that showed task-appropriate discriminative TIA during the interleaved approach training sessions showed significant changes in the approach learning-relevant TIA in correlation with the development of avoidance learning-relevant TIA.

Neuronal activity: excitatory TIA during avoidance learning and activity during the corresponding discriminative approach training sessions

Brief review of the hypotheses

Excitatory TIA is a significantly increased neuronal response during training compared with the response during pretraining 
with unpaired tone and shock presentations. The analysis evaluated the following hypotheses: excitatory TIA will occur during avoidance conditioning; different areas will exhibit maximal or "peak" excitatory TIA in different stages of avoidance learning (see introductory remarks); and the predicted changes in excitatory TIA during avoidance learning will not be found during the corresponding approach training sessions.

\section{Context-specific early training stage excitatory TIA in the} anterior and posterior cingulate cortices

Early excitatory TIA occurred in anterior cingulate cortical area $24 \mathrm{~b}$ and posterior cingulate cortical area $29 \mathrm{c} / \mathrm{d}$ (Figs. 4, 6), as indicated by a significant increase of $\mathrm{CS}^{+}$-clicited ncuronal activity from the pretraining session to the first avoidance conditioning session (area 24b: intervals 19-30, $p<0.05$; area 29c/d: intervals $16-30, p<0.05)$. This TIA persisted into the session of the first discrimination (area 24b: intervals $10-30, p<0.05$; intervals 13 , $16-20,29$, and $30, p<0.05$ ) and the criterial session (area $24 \mathrm{~b}$ : intervals $12-30, p<0.05$; area $29 \mathrm{c} / \mathrm{d}$ : intervals 18 and 28-30, $p<$ 0.05 ). Significant excitatory TIA also occurred in area $29 \mathrm{c} / \mathrm{d}$, but not in area $24 \mathrm{~b}$, during the overtraining session. These changes were context-specific. There were no significant changes of the neuronal response to the $\mathrm{CS}^{+}$during the corresponding approach training sessions, in association with the avoidance-relevant excitatory TIA in area $24 \mathrm{~b}$ and area $29 \mathrm{c} / \mathrm{d}$ (Figs. 4, 6).

\section{Context-specific intermediate training stage excitatory TLA in} the $A V$ thalamic nucleus and hippocampal area $C A I$

The average neuronal records of the AV nucleus (Fig. 5) and hippocampal area CA1 (Fig. 7) exhibited maximal excitatory TIA during the intermediate stages of avoidance learning, i.e., the session of first discrimination and the session of criterion attainment. However, significant excitatory TIA did not occur ir these areas during the first conditioning session or during the overtraining session. The average neuronal response elicited by the $\mathrm{CS}^{+}$ during preliminary training was exceeded significantly by the response elicited during the session of first discrimination (AV: intervals $16-30, p<0.05$ ) and during the criterial session ( $\mathrm{AV}$ nucleus: intervals $18-30, p<0.05$ ). As in the case of the anterior and posterior cingulate cortex, these changes were contextspecific. No significant change in elicited neuronal activity occurred during the corresponding approach training sessions, in association with the advent of avoidance-relevant excitatory TIA in hippocampus or the AV nucleus.

\section{Context-specific late training stage excitatory $T L A$ in the $M D$ thalamic nucleus}

Significant excitatory TIA did not occur in the first conditioning session or in the session of first behavioral discrimination, but was present during the criterial and overtraining sessions (Fig. 3). The average neuronal response to the $\mathrm{CS}^{+}$increased significantly from the pretraining session to the criterial and overtraining sessions (both comparisons, $p<0.05$ ). Again, these changes were contextspecific. There were no corresponding changes during the interleaved approach training sessions.

\section{Single-subject neuronal records and excitatory TIA}

The foregoing results showed stage-related (early, intermediate, and late) peaks of excitatory TIA in the different monitored areas. The collection of training stage-related peaks of excitatory TIA that occur within a given stage of training constitutes what is meant by a topographic pattern. Thus, the peaks are the building blocks of the patterns. It is important to note that the training

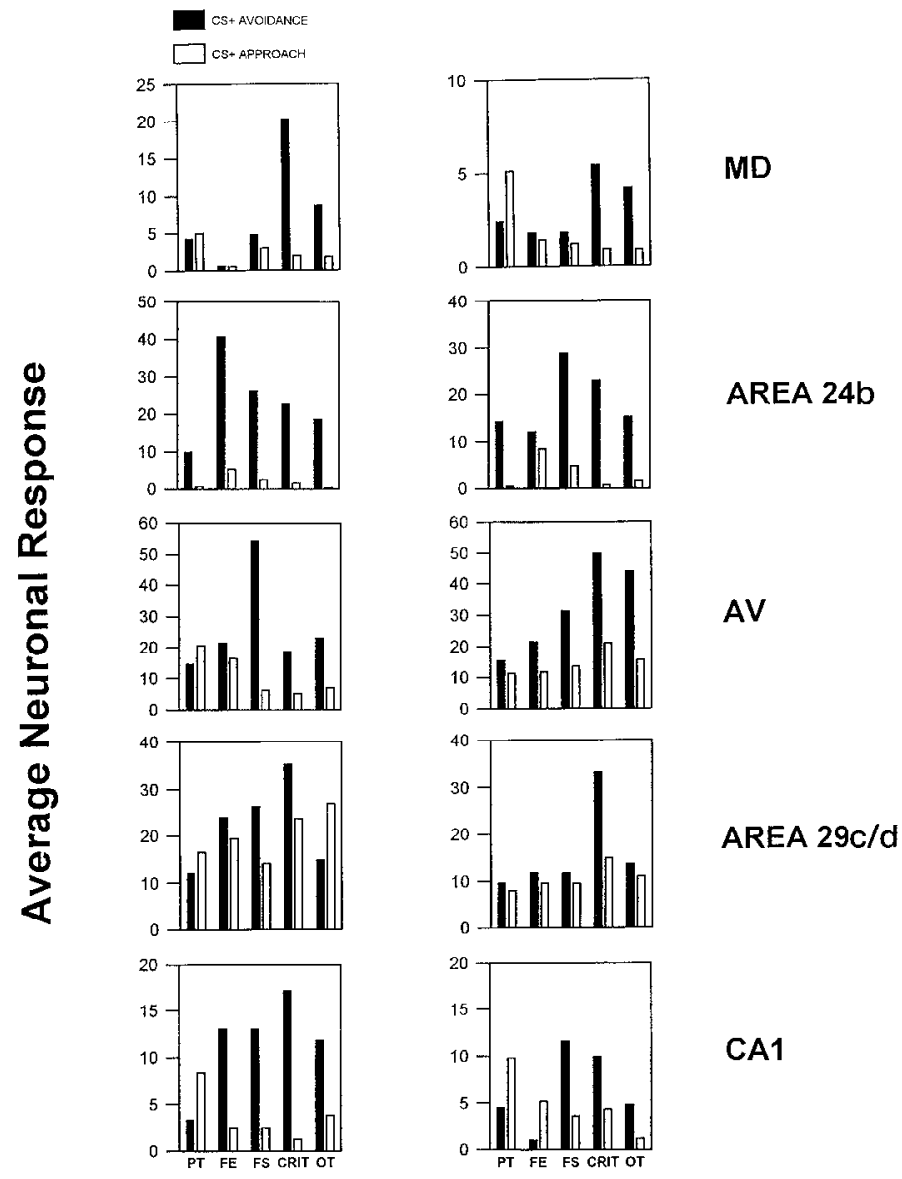

Figure 8. Average integrated unit activity of individual rabbits during the stages of avoidance learning (solid bars) and corresponding overtraining sessions for the approach task (open bars). The data of two subjects are shown for each monitored area: the $M D$ thalamic nucleus (first row), anterior cingulate cortical $A R E A 24 b$ (second row), the $A V$ thalamic nucleus (third row), posterior cingulate cortical $A R E A$ 29c/d (fourth row), and hippocampal area CAI (fifth row). The data are in the form of $z$-scores normalized with respect to a $300 \mathrm{mscc}$ pre-CS baseline. See Figure 2 legend for definitions of avoidance training stages.

stage-related peaks of excitatory TIA found in this study were relatively coarse peaks compared with peaks found in a previous study (Gabriel et al., 1991), which were based on a larger number of neuronal records accumulated over many years. The previous analysis of the accumulated data set showed distinct peaks in different training stages in each of four layers of posterior cingulate cortex and in the different (magnocellular and parvocellular) divisions of the AV nucleus. Moreover, the training stagedependent peaks of TIA in the past study were generally "sharper" (i.e., confined to one or two training stages) than the peaks observed in this study, which were derived from data averaged over a wider array of cytoarchitectural features. The amount of data obtained in this study was insufficient for observation of topographic patterns at a level of cytoarchitectural resolution comparable with that attained in the previous study. It was nevertheless possible to observe in the present data distinct training stage-specific peaks of excitatory TIA in particular nuclei and cortical layers, using the average neuronal responses of individual subjects (Fig. 8, solid bars). These peaks were very similar to peaks of TIA found in the larger study. Juxtaposed plots (open bars) of the average neuronal discharges elicited during the corresponding sessions of approach training indicated, as in the average data of 
this study (above), the context specificity of the training stagerelated peaks of excitatory TIA. Again, the individual peaks of TIA were context-specific. As shown in Figure 8, the dramatic avoidance learning stage-related peaks of excitatory TIA were not evident in the individual records during the corresponding approach training sessions.

\section{Consistent task-related differences in the magnitude of excitatory $T L A$}

The overall magnitude of excitatory TIA was significantly greater, in a majority of monitored areas, during avoidance training compared with discharges during performance in the corresponding approach training sessions. These differences can be seen by comparing the magnitudes of the average plotted neuronal responses in Figures 3-7 (top vs bottom panels). These effects were indicated by simple effect tests that compared the average $\mathrm{CS}^{+}$related neuronal response during avoidance training with the average $\mathrm{CS}^{+}$-related neuronal response during corresponding approach training sessions. This difference was evident in terms of comparisons of the $\mathrm{CS}^{+}$-related discharges (MD: overtraining, $p<0.05$; area 24b: first significant discrimination, intervals $12-30$, and criterion, intervals $1230, p<0.05$; $\mathrm{AV}$ : first significant discrimination, intervals $13-30$, criterion, intervals $14-30$, and overtraining, intervals $16-30, p<0.01$; area $29 \mathrm{c} / \mathrm{d}$ : first session, intervals $11-30$, first significant discrimination, intervals $11-30$, criterion, intervals $15-30$, and overtraining, intervals $12-30, p<$ $0.05)$. Differences in the overall magnitude of excitatory TIA in hippocampal area CA1 (Fig. 7) were not significant.

\section{DISCUSSION}

In this study, discriminative instrumental approach training of rabbits to asymptotic levels of performance was followed by discriminative instrumental avoidance training. The same pure-tone conditioned stimuli were used for both tasks, but the assignment of tones as $\mathrm{CS}^{+}$and $\mathrm{CS}^{-}$was reversed. Avoidance training sessions were alternated with continuing sessions of training in the well learned approach task, one session each day. The basic question was: would the pronounced training-induced changes in tone-elicited excitation and neuronal discrimination that have been shown to occur in cingulate cortex, limbic thalamus, and hippocampus during avoidance learning carry over and be evidenced during the concurrent approach training sessions? The alternative possibility was that the neuronal changes would be context-specific: the changes during avoidance learning would take place only in the avoidance training situation, whereas the same physical stimuli would elicit the very different, approach training-relevant neuronal activity during the concurrent approach training sessions.

All of the monitored areas developed significant discriminative TIA (greater discharges in response to the $\mathrm{CS}^{+}$than to the $\mathrm{CS}^{-}$) during approach learning. The same neurons in response to the same physical stimuli developed discriminative TIA appropriate to the avoidance task, while showing approach learning-related discriminative TIA during the concurrent performance of the well learned approach task (except for the MD nucleus). The exhibition of task-appropriate "reversed" discriminative discharges in the two tasks is unprecedented and constitutes a clear demonstration of context specificity of the training-induced neuronal discrimination.

A remarkable feature of the learning-related neuronal firing in cingulate cortex and limbic thalamus is the occurrence of distinct topographic patterns of CS-elicited excitation in different stages of hehavioral acquisition (Gabriel et al., 1991). This study also shows context specificity of the topographic patterns. Such patterns occurred in the present study as expected, during avoidance training. However, no corresponding topographic patterns were found during the concurrent approach training sessions. Instead, the records of each area during the concurrent approach training sessions showed an excitatory discharge that did not change significantly over sessions.

The training stage-related topographic patterns of excitatory TIA are hypothesized to represent a neural code of the spatiotemporal context that defines a particular learning situation (Gabriel et al., 1991, 1993). This hypothesis specifies that two conditions must be met to observe a particular topographic pattern in the brain: (1) a particular spatial circumstance (the rabbits must be in a specific training apparatus), and (2) a particular temporal circumstance (a specific stage of behavioral learning, e.g., initial, intermediate, asymptotic, overlearned, etc.). The present data provide direct support for the spatial context specificity of the topographic patterns (i.e., condition 1 above). Before this study, empirical evidence of the spatial context specificity of the topographic patterns was not available because testing had been carried out only in the avoidance training situation. Temporal context specificity is given by virtue of the changing topography over training stages. Studies to establish whether the changes of the topography during training are related to the passage of time per se or to the accumulation of task experience are currently in progress.

The theoretical position evaluated here states further that the topographic patterns are involved in mediating context-specific memory retrieval. A particular topographic pattern of neural excitation, induced by a particular training context as input, may in turn educe a particular response or memory as output. Because the topographic patterns occur only in particular spatio-temporal contexts, the induction of a particular pattern is unlikely to call forth memories and/or behaviors associated with other learning contexts. Thus, the patterns could reflect the operation of a dynamic neural mechanism for memory retrieval with minimal proactive and/or retroactive interference (failure to remember and respond appropriately because of intrusion of memories/ responses acquired in other contexts). Interference-free retrieval is widely recognized as the principal problem confronting the design of memory systems (McClelland et al., 1986). The efficacy of contextual information for minimizing interference has been well recognized in the literature of animal and human memory (l'ulving and 'Ihomson, 1973; Bouton, 1990; Gordon and Klein, 1994).

Scveral lines of cvidence support an involvement of the topographic patterns in recall. First, the remarkable stability of context-appropriate behavior in the avoidance and approach tasks is mirrored by the exquisite context sensitivity shown here at the level of the neuronal activity. The rabbits performed each task as if it were the only task being engaged, i.e., no indication of intertask interference was found in the behavioral data. Second, it has been noted that hippocampal formation lesions interfere with the topographic patterns (Gabriel et al., 1987), suggesting that the topography-producing dynamics of the circuitry depend on influences that flow via well known pathways from the hippocampal formation to the cingulate cortex and limbic diencephalon. The disturbance of the patterns in rabbits with hippocampal formation lesions, therefore, would be expected to produce context insensitivity at the behavioral level. Such insensitivity was indicated by the lack of response to an altered context in rabbits with hip- 
pocampal lesions (Kang et al, 1990). These results are in accord with studies using other conditioning paradigms, which indicate an important role of the hippocampal formation in the mediation of contextual control of behavior (Winocur et al., 1987; Penick and Solomon, 1991; Phillips and LeDoux, 1992; Kim et al., 1993; McAlonan et al., 1995). Finally, consistent with a hippocampal involvement in neuronal and behavioral context specificity, area $\mathrm{C} \wedge 1$ neurons were the only neurons monitored in this study that exhibited complete context specificity, i.e., no carryover of the approach training-relevant neuronal discrimination to the pretraining session in the avoidance training apparatus.

The present interpretation suggests that the occurrence of different topographic patterns in different training situations is associated with interference-free recall and performance. However, the data did not show different topographic patterns in the approach and avoidance training situations. Instead, they showed the clear presence of topographic patterns at various stages of avoidance training and the absence of topographic patterns during the concurrent sessions of approach training. Thus, the actual contrast in this study was between the presence versus the absence of patterns, respectively, in the two tasks.

The neuronal populations in limbic thalamus and cingulate cortex monitored during acquisition of the avoidance and approach habits all showed stage-specific peaks of excitatory TIA, i.e., first a rise, followed with further training by a decline, of excitatory TIA magnitude. Different areas exhibited peaks of TIA in different stages of behavioral acquisition. However, the fact that the magnitude of TIA eventually declined in all of the areas suggested that the final state of excitatory TIA in a highly overtrained subject is low in magnitude, with little or no topography. This situation describes the activity recorded in the present study during the concurrent sessions of approach training, which on average represented the 14 th through 18 th sessions of training in that task. The final, "stable" state of the system, indicated by the low level of TIA during these sessions, may be an indication of noninvolvement of the circuitry in task mediation. Consistent with this hypothesis, limbic thalamic lesions, which essentially blocked acquisition of the avoidance behavior, had little impact on retention performance of rabbits given lesions after substantial overtraining (Hart et al., 1995). The implication of these findings is that other as yet unidentified circuitry is involved in task mediation in the highly overtrained state.

These considerations relate the dynamic time-dependent changes of TIA during the progress of approach and avoidance learning to changes that may underlie memory consolidation, as assessed in other learning paradigms (Squire et al., 1984; Alvarez and Squire, 1994). Viewed in this way, the present data suggest a function of the time-dependent changes that is not usually considered in discussions of consolidation processes: the timedependent changes may have as their principle function the production of situation-specific spatio-temporal patterns of neural excitation that code the learning context and promote interference-free recall.

It is important to note that the topographic patterns found in the present study were based on peaks of TIA in a wider array of areas (including anterior cingulate cortex, the MD thalamic nucleus, and hippocampal area CA1) than in a previous study, which assessed TIA in only posterior cingulate cortex and the anterior thalamic nuclei (Gabriel et al., 1991). Whereas the peaks in all of these areas could constitute, as proposed, a neural retrieval code in the brain, it is not likely that all of these areas act in coordination in the service of a single mnemonic process. Rather, compelling evidence exists in support of the hypothesis that anterior cingulate cortex and related thalamic areas (the "anterior circuit") subserve a different mnemonic process than that subserved by the "posterior circuit," consisting of posterior cingulate cortex and related thalamic areas (Gabriel, 1990).

We propose that the anterior circuit is involved in mediating working or recency memory, a high-capacity memory system that encodes new inputs rapidly on first presentation and holds them in store for immediate use. Persistence of material in this system is limited, because encoded material is likely to be displaced by newer inputs and/or to decay rapidly. In contrast, storage in the posterior circuit is involved in reference or primacy memory, which develops gradually and incrementally and thus requires repetition of inputs or rehearsal. Because of the need for repetition, it is not new material that is stored in the posterior circuitrather, it is repetitive regularities encountered in the learning situation. Features of a situation that have been encountered often or rehearsed sufficiently to be encoded in the posterior circuit endure even after those features change. New features are encoded in the anterior circuit while the posterior circuit retains the older features and contingencies, at least for some time, until repetition of the new features gradually removes the older features.

This distinction is based on many observations (Gabriel, 1990). For example, neurons in the anterior (working memory) circuit at cortical and thalamic levels discriminated between $\mathrm{CS}^{+}$and $\mathrm{CS}^{-}$ more rapidly (after fewer trials and at briefer latencies) during CR acquisition than neurons in the posterior (reference memory) circuit. However, when trained subjects received reversal training, neuronal discrimination between $\mathrm{CS}^{+}$and $\mathrm{CS}^{-}$in the anterior circuit reversed, whereas posterior circuit neurons exhibited the discrimination appropriate to original ("primary") learning throughout reversal training.

The present study indicates an additional finding that is compellingly supportive of the foregoing distinction. Robust discriminative TIA appropriate to the approach habit was evident during the concurrent approach training sessions only in the neuronal records of the posterior circuit (posterior cingulate cortex and the AV thalamic nucleus). As expected of a reference/primacy memory, the discriminative TIA in these structures endured in the concurrent sessions despite the ongoing and incompatible coding of the stimuli for avoidance learning. In contrast, neurons in a structure of the anterior circuit (the MD thalamic nucleus) showed results expected of a working memory: a loss, during the concurrent approach training sessions, of discriminative TIA acquired during the acquisition phase of the approach task. The marginal discriminative TIA in the MD nucleus during the concurrent approach training sessions did not achieve statistically significant levels in any of those sessions.

How is it possible to reconcile the functional distinction between a recency/working memory and a primacy/reference memory with the broad-based attribution of spatio-temporal coding to all of the areas that produce topographic patterns, including anterior and posterior cingulate and the related thalamic areas? We propose that the topographic neural code formed in the anterior circuit for working memory is involved in context-based retrieval for relatively brief time intervals (e.g., minutes to hours). The operation of this circuit is likely to contribute importantly in tasks that require subjects to make relatively short-term mnemonic temporal discriminations (discrimination based on time since an item was stored) (Kesner et al., 1984; Chiba et al., 1994; Kesner et al., 1994). In contrast, the topographic neural code 
formed in the posterior reference memory or mnemonic primacy circuit is relevant to habit age discriminations over a greater time domain (days to weeks). Remarkably, exactly this kind of deficit in human patients has been labeled "retrosplenial amnesia" because of its association with posterior cingulate cortical pathology (Valenstein et al., 1987).

\section{REFERENCES}

Alvarez P, Squire LR (1994) Memory consolidation and the medial temporal lobe: a simple network model. Proc Natl Acad Sci 91:7041-7045.

Bouton ME (1990) Contexts and retrieval in extinction and in other examples of interference in simple associative learning. In: Current topics in animal learning: brain, emotion, and cognition (Dachowski LW, Flaherty CF, eds), pp 25-53. Hillsdale, NJ: Erlbaum.

Brogden WJ, Culler FA (1936) A device for motor conditioning of small animals (Abstr). Science 83:269A.

Buchwald J, Holstein SB, Weber DS (1973) Multiple unit recording: technique, interpretation, and experimental applications. In: Bioelectric recording techniques, Part $\mathrm{A}$, Cellular processes and brain potentials (Thompson RF, Patterson MM, eds), pp 202-242. New York: Academic

Chiba AA, Kesner RP, Reynolds AM (1994) Memory for spatial location as a function of temporal lag in rats: role of hippocampus and medial prefrontal cortex. Behav Neural Biol 61:123-131.

Donovick PJ (1974) A metachromatic stain for neural tissue. Stain Technol 49:49-51.

Fox CA, Eichman J (1959) A rapid method for locating intracerebral clectrode tracks. Stain Technol 34:39-42.

Gabriel M (1990) Functions of anterior and posterior cingulate cortex during avoidance learning in rabbits. The prefrontal cortex: its structure, function and pathology. Prog Brain Res 85:467-483.

Gabriel M (1993) Discriminative avoidance learning: a model system. In: Neurobiology of cingulate cortex and limbic thalamus: a comprehensive handbook (Vogt BA, Gabriel M, eds), pp 478-523. Boston: Birkhauser.

Gabriel M, Flannery K, Cuppernell C (1993) Context-specific and context-general multi-site limbic neuronal activity during concurrent approach and avoidance training. Soc Neurosci Abstr 19:802.

Gabriel M, Foster K, Orona E, Saltwick SE, Stanton M (1980) Neuronal activity of cingulate cortex, anteroventral thalamus and hippocampal formation in discriminative conditioning: encoding and extraction of the significance of conditional stimuli. In: Progress in physiological psychology and psychobiology, Vol 9 (Spraguc J, Epstcin AN, cds), pp 126-223. New York: Academic.

Gabriel M, Hart M, Poremba A (1995) Prediction of learned behavior from electrophysiology: diminished contribution of limbic thalamus to avoidance responding after extensive overtraining in rabbits. Soc Neurosci Abstr 21:1930.

Gabriel M, Kubota Y, Sparenborg S, Straube K, Vogt BA (1991) Effects of cingulate cortical lesions on avoidance learning and training-induced unit activity in rabbits. Exp Brain Res 86:585-600.

Gabriel M, Sparenborg S, Stolar N (1987) Hippocampal control of cingulate cortical and anterior thalamic information processing during learning in rabbits. Exp Brain Res 67:131-152.
Gabriel M, Vogt VA, Kubota Y, Poremba A, Kang E (1991) Trainingstage related neuronal plasticity in limbic thalamus and cingulate cortex during learning: a possible key to mnemonic retrieval. Behav Brain Res 46:175-185.

Gerhard L (1968) Atlas of the mes- and diencephalon of the rabbit. Berlin: Springer.

Girgis M, Shih-Chang W (1981) A new stereotaxic atlas of the rabbit brain. St. Louis: Green.

Gordon WC, Klein RL (1994) The effects of context change on retention performance. In: Animal learning and cognition (Mackintosh N, ed), pp 255-279. New York: Academic.

Hirsh R (1974) The hippocampus and contextual retrieval of information from memory: a theory. Behav Biol 12:421-444.

Huynh H, Feldt LS (1976) Estimation of the Box correction for degrees of freedom from sample data in randomized block and split-plot designs. J Educat Stat 1:69-82.

Kang E, Kubota Y, Poremba A, Gabriel M (1990) Hippocampal lesions, limbic cortical and thalamic training-induced unit activity, avoidance conditioning, and response to different forms of novelty in rabbits. Soc Neurosci Abstr 16:264.

Kesner RP, Chiba AA, Jackson-Smith P (1994) Rats do show primacy and recency effects in memory for lists of spatial locations: a reply to Gaffan. Animal Learn Behav 22:214-218.

Kesner RP, Measom MO, Forsman SL, Holbrook TH (1984) Serialposition curves in rats: order memory for episodic spatial events. Animal Learn Behav 12:378-382.

Kim JJ, Rison RA, Fanselow MS (1993) Effects of amygdala, hippocampus, and periaqueductal gray lesions on short- and long-term contextual fear. Behav Neurosci 107:1093-1098.

McAlonan GM, Wilkinson LS, Robbins TW, Everitt BJ (1995) The effects of AMPA-induced lesions of the septohippocampal cholinergic projection on aversive conditioning to explicit and contextual cues and spatial learning in the water maze. Eur J Neurosci 7:281-292.

McClelland JL, Rumelhart DE, Hinton GE (1986) The appeal of parallel distributed processing. In: Parallel distributed processing, Vol 1 (Rumelhart DE, McClelland JL, eds), pp 3-44. Cambridge: MIT.

Nadel L, Willner J (1980) Context and conditioning: a place for space. Physiol Psychol 8:218-228.

Penick S, Solomon PR (1991) Hippocampus, context, and conditioning. Behav Neurosci 105:611-617.

Phillips RG, LeDoux JE (1992) Differential contribution of amygdala and hippocampus to cued and contextual fear conditioning. Behav Neurosci 106:274-285.

Squire LR, Cohen NJ, Nadel L (1984) The medial temporal region and memory consolidation: a new hypothesis. In: Memory consolidation (Weingarner H, Parker E, eds), pp 185-210. Hillsdale, NJ: Erlbaum.

Tulving. E, Thomson DM (1973) Encoding specificity and retrieval processes in episodic memory. Psychol Rev 80:352-373.

Valenstein E, Bowers D, Verfaellie M, Heilman KM, Day A, Watson R'I (1987) Retrosplenial amnesia. Brain 110:1631-1646.

Winer BJ (1962) Statistical principles in experimental design. New York: McGraw-Hill.

Winocur G, Rawlins JNP, Gray JA (1987) The hippocampus and conditioning to contextual cues. Behav Neurosci 101:617-625. 